\title{
Anchoring Risk Analysis and Management of Anchored Vessels at Tangshan Caofeidian Port
}

\author{
Feng Wang ${ }^{1,2}$, Qiang Long ${ }^{1}$, Zhengjiao $\mathrm{Qi}^{3}$, Chang Wang ${ }^{1}$, Yi Lin ${ }^{4}$ \\ ${ }^{1}$ Caofeidian Meteorological Bureau, Hebei Tangshan, Tangshan 063015, China \\ ${ }^{2}$ Tangshan Meteorological Bureau,Tangshan 063000, China \\ ${ }^{3}$ Caofeidian Maritime Bureau, Tangshan 063015, China \\ ${ }^{4}$ Meteorological Institute, Tianjin Meteorological Bureau, Tianjin 300074, China
}

\section{唐山曹妃甸港针泊船走针的风险分析及管理}

\author{
王锋 ${ }^{1,2}$, 龙强 ${ }^{1}$, 齐正胶 ${ }^{3}$, 王畅 ${ }^{1}$, 林毅 ${ }^{4}$ \\ ${ }^{1}$ 唐山市曹妃甸区气象局, 唐山 063015 , 中国 \\ 2 唐山市气象局, 唐山 063000 , 中国 \\ 3 唐山曹妃甸海事局, 唐山 063015, 中国 \\ ${ }^{4}$ 天津市气象局气象科学研究所, 天津 300074 , 中国
}

\begin{abstract}
Using the fuzzy comprehensive evaluation method based on AHP, combined with the maximum degree of membership and the subjective scoring of influencing factors, the risk of mooring ships anchoring in the Caofeidian harbor anchorage was analyzed, and the weights of selected influencing factors were determined, and in response to the maritime and meteorological business processes, the time node, key links, and important details of anchoring risk control are emphasized. The results show that the anchoring risk of the Caofeidian anchorage is very high. Among the selected objective factors, wind power is the main factor which leads to anchor removal, followed by traffic density. The management and control of the anchoring risk needs the cooperation of the
\end{abstract}

基金项目：环渤海区域科技协同创新基金项目 (QYXM201609); 唐山市气象局课题项目

(tssqx j201701)

作者简介: 王锋 (1962-), 男, 高级工程师, 主要从

事海洋气象服务管理工作。E-mai1:tsqx_fw@163.com meteorological and maritime departments, and the refinement the various risk links, so as to reduce the occurrence of ship anchor accidents effectively.

Keywords: dragging anchor; risk; AHP; evaluation; management

\section{摘要}

利用基于层次分析的模糊综合评价方 法, 结合最大隶属度原则、影响要素的主 观打分, 分析了唐山曹妃甸港针地针泊船 走针的风险, 确定了所选影响因素的权重, 并针对海事、气象业务流程, 指出了走针 风险管控所须关注的时间节点、关键环节 和重要细节等内容。结果表明: 唐山曹妃 甸港针地的船舶走针风险很高, 在所选的 客观因素中, 风力是导致走针的最主要因 素, 其次是交通密度。走针风险的管控需 要气象、海事部门的共同协作，细化各个 风险环节，才能有效减少船舶走针事故的 发生。

关键词：走针；风险；层次分析；评价； 管理 


\section{1. 引言}

针地针泊船的监管是各船舶交通管理 中心的一项重要业务内容 ${ }^{[1]}$, 特别是随着海 洋、港口经济快速的发展, 走针事故频发 ${ }^{[2-}$ ${ }^{4]}$, 各地交管中心高度重视针地针泊作业和 走针风险管控。目前针泊业务管理大多依 赖于个人经验、实时不断的人为监控以及 严格的值班制度。然而, 由于值班人员的 阅历、认识、经验以及反应能力等不尽相 同, 对恶劣天气下针泊船的走针风险认识 程度不一, 且针泊船的安全管理目前还没 有明确的监管标准, 针泊作业存在着较大 的环境不确定性, 以及由此所致的潜在走 针风险。影响针泊船安全针泊的因素很多, 如风、浪、流以及针地底质、针泊船密度 等客观因素, 也有针泊方式、应急处置等 主观因素。根据多年的海洋气象预报服务 经验积累和需求调研反馈, 我们认为这些 因素应该有主次、轻重之分, 也就是说, 各因素在导致针泊船走针的整体评估中应 该有各自的影响权重。为了确定这一问题, 科学、定量的观测、实验以及足够的案例 积累十分必要, 但限于成本、机制、信息 不对称等原因, 难以实现十分精确的判断 和分析。

因此, 针对防灾减灾和风险预估的需 求, 基于主观评价并采用一定方法的风险 分析就显得十分必要。文中利用基于层次 分析的模糊综合评价方法 ${ }^{[5-8]}$ 、隶属度判断 以及调查研究、分值匹配等, 对曹妃甸港 针地针泊船走针的风险高低、主要影响因 素作了分析, 并结合气象、海事的业务流 程特征, 指出了风险管控所须关注的时间 节点、关键环节和重要细节内容。

\section{2 曹妃甸港针地特征}

曹妃甸港外针地共有 2 处, 分为东针 地和西针地, 位置如图 1 所示。西针地为 港口综合性针地, 面积约 65 平方公里, 自 然水深 12 米至 29 米, 东南部为大型油船 针地, 水深较大; 东针地主要供大型散货 船、原油船、LNG 船等针泊, 面积约 128 平 方公里, 自然水深 21 米至 32 米, 西北角 水域为液化天然气 (LNG) 船舶应急针地。
针地的底质构成对针落水抓力的发挥 具有重要影响 ${ }^{[9]}$, 一般来说, 软硬适度的沙 地、粘土质海底的针地较好, 沙泥混合的 海底次之, 而硬质泥沙的底质较差。曹妃 甸港周边海域沉积物主要为淤泥、粉砂和 黏土等, 约占总沉积物的 $85 \%$, 针地底质 基本均为硬泥。可见, 曹妃甸针地的底质 并不理想, 在一定程度上也增加了针泊船 走针的风险。

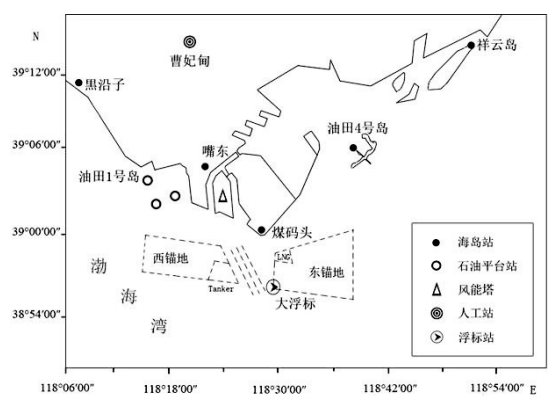

图 1 针地地理位置及海洋气象观测站点分布图

根据近四年的走针案例统计, 曹妃甸 港外针地年均出现较严重的走针事故 4-5 例, 走针的船舶尺寸大小不一、载重各不 相同, 事故发生的地理位置也不一样。

\section{3 针泊船走针风险分析}

文中采用基于层次分析法的模糊综合 评价方法分析京唐港针泊船的走针风险, 确定主要影响因素的权重。按照方法原理 和研究对象, 确定本模型综合评价的对象

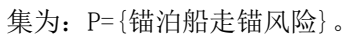

\section{1 评价指标}

影响船舶走针的安全因素很多, 根据 船舶交通管理中心的工作经验和业务需求, 所选取的影响因子 (即评价指标) 应该对 针泊船走针的影响较大, 这样得到的分析 结论更有针对性和可用性。根据相关学者 的分析, 在针地基本参数 (大小、位置、 底质等) 确定的条件下, 诸多影响船舶走 针的因素中, 针地风力大小、交通密度、 针泊船尺寸、船型是重要的客观指标, 船 员的综合素质、船长的应急能力为主观因 
素, 无法统一。因此, 在交通管理中心了 解本地针地情况、熟悉通航业务的基础上, 确定评价指标为: 风力、交通密度、船舶 尺寸和船型。

（1）风力。根据《河北省灾害性天气 预警信号与防御指南》对大风预警信号的 规定, 当渤海海区平均风力达 $7 \sim 8$ 级, 或 阵风 9 级以上发布蓝色预警信号, 平均风 力达 9 10 级, 或阵风 11 级以上发布黄色 预警信号, 平均风力达 $11 \sim 12$ 级, 或阵风 13 级以上发布橙色预警信号, 平均风力达 12 级以上发布红色预警信号。根据该规定, 风力指标确定为: 7 级 (阵风 9 级)、9 级 (阵风 11 级)、 11 级 (阵风 13 级) 13 级 及以上四个指标。

(2) 交通密度。交通密度是指针地针 泊船的流量, 密度小时直接于针地抛针, 密度中等时, 船舶需要在针地内进行必要 的疏散, 密度大时就需要针地外抛针。因 此, 交通密度也是影响针泊的重要因素, 分为密度小、密度中等、密度大三个指标。

(3) 船舶尺寸。根据船舶的尺寸确定 抛针地点是提高针地利用效率的基础, 对 船舶安全针泊、保证针泊秩序也十分有利。 根据曹妃甸港的通航实际和业务需求, 将 船舶分为小型 (船长 130 米以下)、中型 (船长 130-200 米)、大型 (船长 200-280 米) 和超大型 (船长 280 米以上) 四类。

(4) 船型。根据船舶对针泊的条件要 求, 将船型分为: 一般船舶、客船类、危 险品船和特殊船类。一般船舶对针地没有 特殊要求; 客船涉及到承载人员, 所以安 全性级别要求很高; 危险品船包括油轮、 液化天然气货轮等运载易燃、易爆货物的 船舶, 有的需要专门的针地。

\section{2 指标集和评价集}

根据评价指标分析, 确定指标集合为: $\mathrm{U}=\left\{u_{1}, u_{2}, u_{3}, u_{4}\right\}=\{$ 风力大小、交通密度、

船舶尺寸、船型\}（1）

各指标的二级评价指标为:
表 1 针泊船走针风险评价指标集

\begin{tabular}{|c|c|c|c|}
\hline \multicolumn{2}{|c|}{$\begin{array}{l}\text { 一级评 } \\
\text { 价指标 }\end{array}$} & \multicolumn{2}{|r|}{ 二级评价指标 } \\
\hline \multirow{4}{*}{$u_{1}$} & \multirow{4}{*}{$\begin{array}{l}\text { 风 } \\
\text { 力 } \\
\text { 大 } \\
\text { 小 }\end{array}$} & $u_{11}$ & $\begin{array}{l}\text { 海区平均风力 } 7 \text { 级或阵 } \\
\text { 风 } 9 \text { 级以上 }\end{array}$ \\
\hline & & $u_{12}$ & $\begin{array}{l}\text { 海区平均风力 } 9 \text { 级或阵 } \\
\text { 风 } 11 \text { 级以上 }\end{array}$ \\
\hline & & $u_{13}$ & $\begin{array}{l}\text { 海区平均风力 } 11 \text { 级或阵 } \\
\text { 风 } 13 \text { 级以上 }\end{array}$ \\
\hline & & $U_{14}$ & $\begin{array}{l}\text { 海区平均风力 } 13 \text { 级及以 } \\
\text { 上 }\end{array}$ \\
\hline \multirow{3}{*}{$u_{2}$} & \multirow{3}{*}{$\begin{array}{l}\text { 交 } \\
\text { 通 } \\
\text { 密 } \\
\text { 度 }\end{array}$} & $U_{21}$ & 密度小 \\
\hline & & $u_{22}$ & 密度中等 \\
\hline & & $u_{23}$ & 密度大 \\
\hline \multirow{4}{*}{$u_{3}$} & \multirow{4}{*}{$\begin{array}{l}\text { 船 } \\
\text { 舶 } \\
\text { 尺 }\end{array}$} & $u_{31}$ & $\begin{array}{l}\text { 小型（船长 } 130 \text { 米以 } \\
\text { 下) }\end{array}$ \\
\hline & & $U_{32}$ & $\begin{array}{l}\text { 中型（船长 130-200 } \\
\text { 米) }\end{array}$ \\
\hline & & $U_{33}$ & $\begin{array}{l}\text { 大型（船长 200-280 } \\
\text { 米) }\end{array}$ \\
\hline & & $U_{34}$ & $\begin{array}{l}\text { 超大型（船长 } 280 \text { 米以 } \\
\text { 上） }\end{array}$ \\
\hline \multirow{4}{*}{$u_{4}$} & \multirow{4}{*}{$\begin{array}{l}\text { 船 } \\
\text { 型 }\end{array}$} & $u_{41}$ & 一般船舶 \\
\hline & & $u_{42}$ & 客船类 \\
\hline & & $U_{43}$ & 危险品船 \\
\hline & & $U_{44}$ & 特殊船类 \\
\hline
\end{tabular}

按照评价原理和业务应用需求, 确定 评语集为:

$$
\begin{aligned}
& V=\left\{\boldsymbol{V}_{1}, \boldsymbol{V}_{2}, \boldsymbol{V}_{3}, \boldsymbol{V}_{4}\right\}= \\
& \quad\{\text { 很高, 高, 较高, 低 }\}
\end{aligned}
$$

\section{3 评价指标的权向量}

利用层次分析法确定评价指标权向量。

（1）指标权重

通过调研确定四个评价指标的相对重 要性, 结果如表 2 所示。

\begin{tabular}{cccccc}
\multicolumn{7}{c}{ 表 2 四个一级指标的调研判断 } \\
\hline $\mathrm{U}$ & $u_{1}$ & $U_{2}$ & $U_{3}$ & $u_{4}$ & 权重 \\
\hline$u_{1}$ & 1 & 2 & 7 & 4 & $W_{1}$ \\
\hline$u_{2}$ & $1 / 2$ & 1 & 4 & 3 & $W_{2}$ \\
\hline$u_{3}$ & $1 / 7$ & $1 / 4$ & 1 & 2 & $W_{3}$ \\
\hline$u_{4}$ & $1 / 4$ & $1 / 3$ & $1 / 2$ & 1 & $W_{4}$ \\
\hline
\end{tabular}

判断矩阵即为, 


$$
A=\left[\begin{array}{cccc}
1 & 2 & 7 & 4 \\
1 / 2 & 1 & 4 & 3 \\
1 / 7 & 1 / 4 & 1 & 2 \\
1 / 4 & 1 / 3 & 1 / 2 & 1
\end{array}\right] \text { (3) }
$$

此处采用和积法对判断矩阵进行特征 向量计算, 首先计算矩阵各列的和, 然后 按列作归一化处理, 结果如表 3 所示。

表 3 归一化处理和权重结果

\begin{tabular}{lccrrc}
\hline $\mathrm{U}$ & $u_{1}$ & $u_{2}$ & $u_{3}$ & $u_{4}$ & 权重 \\
\hline$u_{1}$ & $J .5283$ & 0.5581 & 0.56 & 0.4 & 2.0464 \\
\hline$u_{2}$ & $J .2642$ & 0.2791 & 0.32 & 0.3 & 1.1633 \\
\hline$u_{3}$ & $J .0755$ & 0.0698 & 0.08 & 0.2 & 0.4253 \\
\hline$u_{4}$ & $J .1321$ & 0.0930 & 0.04 & 0.1 & 0.3651 \\
\hline
\end{tabular}

对判断矩阵 $A$ 进行一致性检验, $A$ 的 最大特征根为:

$$
\begin{gathered}
{\left[\begin{array}{cccc}
1 & 2 & 7 & 4 \\
1 / 2 & 1 & 4 & 3 \\
1 / 7 & 1 / 4 & 1 & 2 \\
1 / 4 & 1 / 3 & 1 / 2 & 1
\end{array}\right]\left[\begin{array}{l}
2.0464 \\
1.1633 \\
0.4253 \\
0.3651
\end{array}\right]=} \\
{\left[\begin{array}{cccc}
\lambda_{1} & 0 & 0 & 0 \\
0 & \lambda_{2} & 0 & 0 \\
0 & 0 & \lambda_{3} & 0 \\
0 & 0 & 0 & \lambda_{4}
\end{array}\right]\left[\begin{array}{l}
2.0464 \\
1.1633 \\
0.4253 \\
0.3651
\end{array}\right]}
\end{gathered}
$$

由上式求得: $\lambda_{1}=4.3054, \lambda_{2}=4.2835$, $\lambda_{3}=4.0879, \lambda_{4}=4.0459$, 可得 $\lambda_{\max }=4.1806$ 。

检验 $A$ 的一致性指标为: $C . I .=(\lambda$ max $\mathrm{n}) /(\mathrm{n}-1), \lambda$ 比 $\mathrm{n}$ 大得越多, $A$ 的不一致性 就越严重。也就是说, 如果用最大特征值 的特征向量作为被比较因素对某因素影响 程度的权向量, $\lambda_{\text {max }}$ 比 $\mathrm{n}$ 大得越多时, 不一 致性的程度越严重, 判断误差就越大。这 里用一致性比例 C.R. 来度量 $A$ 的一致性。

$$
C . R .=C . I . / R . I \text {. }
$$

式中 $R . I$. 为随机一致性指标。

当 $C . R .=0, A$ 是完全一致性矩阵, $C . R$. $<0.1$ 时, 为满意一致性矩阵, C.R. $>0.1$, $A$ 便不具有一致性。

由上可得: C.R. $=0.0677<0.1$, 所以 $A$ 为满意一致性矩阵, 也就是说各一级指标 权重系数的分配是合理的。因此, $A$ 的特征 向量为:

$$
W=\left(w_{1}, w_{2}, w_{3}, w_{4}\right)=
$$

(2.0464, 1.1633, 0.4253, 0.3651) (6)

归一化处理后即可得一级指标矩阵的 权向量:
$A=(0.5116,0.2908,0.1063,0.0913)(7)$

根据上述方法, 获得二级指标的权向 量为:

$$
\begin{aligned}
& A_{1}=(0.0455,0.0962,0.3340,0.5243) \\
& A_{2}=(0.0832,0.1933,0.7235) \\
& A_{3}=(0.5575,0.2596,0.1123,0.0706) \\
& A_{4}=(0.0802,0.2082,0.2284,0.4832)
\end{aligned}
$$

\section{4 模糊评价关系矩阵}

港航管理部门的调度人员、经验丰富的船 长或船员对二级指标内容进行打分, 对各 打分作平均处理以体现整体意见, 并作归 一化处理, 建立的风力、交通密度、船舶 尺寸、船型模糊评价关系矩阵分别为 $R_{I}$ 、 $R_{2} 、 R_{3} 、 R_{4}:$

$$
\begin{aligned}
R_{1} & =\left[\begin{array}{cccc}
0 & 0 & 0.3 & 0.7 \\
0.1 & 0.4 & 0.5 & 0 \\
0.7 & 0.3 & 0 & 0 \\
0.9 & 0.1 & 0 & 0
\end{array}\right] \quad(8) \\
R_{2} & =\left[\begin{array}{cccc}
0 & 0 & 0.1 & 0.9 \\
0.1 & 0.5 & 0.3 & 0.1 \\
0.7 & 0.2 & 0.1 & 0
\end{array}\right] \quad(9) \\
R_{3} & =\left[\begin{array}{cccc}
0.6 & 0.3 & 0.1 & 0 \\
0.7 & 0.2 & 0.1 & 0 \\
0 & 0.2 & 0.4 & 0.4 \\
0 & 0.1 & 0.3 & 0.6
\end{array}\right](10) \\
R_{4} & =\left[\begin{array}{cccc}
0 & 0.2 & 0.5 & 0.3 \\
0.1 & 0.3 & 0.5 & 0.1 \\
0.2 & 0.5 & 0.2 & 0.1 \\
0.4 & 0.3 & 0.2 & 0.1
\end{array}\right](11)
\end{aligned}
$$

\section{5 模糊评价向量}

模糊评价向量的确定采用 $\mathrm{M}(\wedge, \vee)$ 模糊合成算子, 在运算中能够突出主要因 素。计算风力的评价向量为:

$$
B_{1}=A_{1}{ }^{\circ} R_{1}=
$$

$(0.04555,0.09615,0.3340,0.5243)^{\circ}$

$$
\left[\begin{array}{cccc}
0 & 0 & 0.3 & 0.7 \\
0.1 & 0.4 & 0.5 & 0 \\
0.7 & 0.3 & 0 & 0 \\
0.9 & 0.1 & 0 & 0
\end{array}\right]
$$$$
=(0.5243,0.3,0.09615,0.04555)
$$

归一化后的 $B_{I}=$

(0.5428, 0. 3101, 0. 0995, 0.0472)

根据该方法, 可得交通密度、船舶尺 寸、船型的归一化后的评价向量 B2、B3、 B4 分别为:

$B_{2}=(0.5866,0.1676,0.1620,0.0838)$ 
$B_{3}=(0.5352,0.2492,0.1078,0.1078)$

$B_{4}=(0.3967,0.2976,0.2065,0.0992)$

\section{6 模糊综合评价风险分析}

综上可得评价向量为:

$B=A^{\circ} R=(0.5116,0.2908,0.1063,0.0913)^{\circ}$

$\left[\begin{array}{llll}0.5428 & 0.3101 & 0.0995 & 0.0472 \\ 0.5866 & 0.1676 & 0.1620 & 0.0838\end{array}\right]$

$\left[\begin{array}{llll}0.5866 & 0.1676 & 0.1620 & 0.0838 \\ 0.5352 & 0.2492 & 0.1078 & 0.1078 \\ 0.3967 & 0.2976 & 0.2065 & 0.0992\end{array}\right]=$

$\left[\begin{array}{llll}0.3967 & 0.2976 & 0.2065 & 0.0992\end{array}\right]$

$(0.5116,0.3101,0.1620,0.1063) \quad(13)$

归一化后的 $B=$

( $0.4694,0.2845,0.1486,0.0975$ )

模糊评价的结果显示:

$b_{1}=0.4694=\max \left\{b_{j}\right\}, 1 \leq j \leq 4$ （14）

根据最大隶属度原则, 综合指标对哪 个评价等级的隶属度更高, 就将其所要评 价的目标定为该评价等级。因此, 针地针 泊船走针的风险隶属于第 1 级, 即风险很 高; 在所列的影响因素中, 风力对针泊船 走针的影响程度最高, 其次是交通密度, 也就是说, 风力的大小是影响针地针泊船 走针的最主要因素, 交通密度是重要因素。

\section{4 针泊船走针的风险管理}

通过上述的分析, 大风是影响针泊船 走针的重要因素, 气象部门应当加强海面 大风的时间和空间精细化预报预警, 海事 部门应当制定有效的应对措施, 完善监控 管理标准和风险防控体系, 强化应急处置 能力。本节从气象服务的角度, 结合海事 应急处置流程, 浅析针泊船走针的风险管 理。

\section{1 风险管理的时间节点}

根据大风对针泊船影响的整个过程, 将走针风险的管理时间节点划分为大风来 前、大风过境、大风结束, 再细化各时间 节点重点关注的内容。

\section{(1) 大风来前}

关注每日气象部门提供的预报, 对即 将发生的天气有足够的预知, 并做好如下 工作:

（a）关注滚动预报，获取最新的预报
信息, 确保掌握的信息足够准确;

(b) 通过 VHF 等系统将重要信息发送 至相关船舶, 提醒各船舶做好防范大风的 准备;

(c) 通过 CCTV 视频监控系统、气象 监测网, 严密监控海上天气实况;

（d）做好针泊船的登记信息, 特别是 危化货物运输船、大型船舶的针泊情况要 熟知;

(e) 利用现有技术手段, 如不同风力 对应的走针风险, 评估风险等级;

(f) 做好应急处置的准备工作。

（2）大风过境

(a) 所辖海域已经起风，通过 VHF 等 系统发布实况信息, 提醒做好防范工作;

（b）抽查针泊船值班、应急准确情况;

(c) 严密监控针泊船状态, 确保第一 时间发现、第一时间启动应急;

(d) 如出现走针事故, 应积极按照应 急预案要求配合、参与应急行动。

(3) 大风结束

(a) 有序调度相关船舶起针续航;

(b) 做好总结、反馈, 完善应急预案、 业务流程等。

\section{2 风险管理的重要环节}

根据针泊业务管理流程和经验总结, 走针风险的管理环节包括跟踪预报、风险 评估、发布告警、信息记录、强化监控、 随机抽查、应急处置。

跟踪预报: 气象一海事部门应不断强化 信息沟通、共享机制, 畅通信息互通渠道, 确保气象部门的最新预报能够第一时间被 海事部门接收, 海事部门能够实时查看气 象监测网实况。

风险评估：当海面风力预报较大时, 应根据现有的技术能力, 对可能造成走针 的风险作初步评估, 为应急准备、随机抽 查等工作提供借鉴。

发布告警: 在确认预报内容、风险等 级的基础上, 及时发布大风告警, 提醒相 关船舶做好应急准备工作。

信息记录: 记录好辖区内各船舶的联 系渠道、船舶载货等信息, 并对信息作分 
类、分级规整。

强化监控: 多手段监控针泊船的位置、 出链长度、安全针泊距离等, 及时发现问 题、及时予以解决。

随机抽查: 主要对船舶应对大风天气 的应急值守、准备措施、沟通渠道作随机 抽查, 抽查比例应根据风险等级有所不同、 侧重。

应急处置: 当出现走针事故时, 应快 速做出反应和处置。值班人员通过甚高频 电话直联走针船舶, 按要求采取应对措施, 同时通知下游船舶做好避让、协作等准备。 当情况发展迅速、严重时, 应及时告知搜 救值班处, 按照应急预案等要求开展应急 处置工作。

\section{3 风险管理应关注的细节}

（1）风向。曹妃甸针地独特的地理位 置导致不同的风向所致的走针风险等级不 一, 如东北方向是辽东湾, 偏东方向是渤 海海峡, 直通黄海, 较长的风区、频繁的 大风系统、较大的水深等都使得在其他条 件一致的情况下, 东北到偏东大风所致的 船舶走针的风险较高, 而西北风的下垫面 是陆地, 风区长度较小, 所造成的走针风 险就较低。

（2）交通流。影响因素的权重分析结 果显示, 交通密度是除了风力之外对走针 最有影响的因素, 值班人员应熟悉辖区内 的航线、气象和水文环境、业务内容等, 这样才能有针对性地开展防范工作。

(3) 船员素质。由于工作年限、认知 能力、学习深度、阅历积累等不同, 各船 舶船员的综合素质往往也不一样, 对工作 的职业操守、责任心也参差不齐, 这就导 致了同样的指令被实施的结果、程度也可 能不一样。因此, 值班、调度等管理人员 应考虑该因素, 加强抽查、沟通, 管控不 必要的风险。

\section{5 总结}

（1）模糊综合评价方法计算和隶属度 分析的结果表明, 唐山曹妃甸港针地的针
泊船走针的风险很高。

（2）在所选定的影响因子中, 风力是 导致针泊船走针的最主要因素, 其次是交 通密度, 船舶尺寸、船型因素的作用明显 偏小。

(3) 针泊船走针的风险管控需要气象、 海事等部门的有效协作, 从安全管理的时 间节点、关注环节和重要细节入手, 才能 减少大风天气条件下险情和事故的发生。

\section{参考文献}

[1] 赵亮. 基于模糊故障树方法的船舶针泊 安全分析.大连海事大学,2017.

[2] 吴卫兵,尹建川.准确判断船舶走针方法. 中国航海,2012,35(04):124-127.

[3] 刘勇, 徐海军, 李振. 针泊船舶的安全警戒 范围.航海技术,2015(05):31-33.

[4] 祝贵兵,池弘福,吴剑锋. 灾害天气下无动 力船针泊安全性分析. 浙江海洋学院学 报(自然科学版),2012,31(01):70-74.

[5] Kaufman A, Gupta M M. Int reduction to fuzzyarithmetic: theory and applications. New York: Van Nostrand Reinhold,1985.

[6] Zhu W, You Q J. 2016. High-rise Buildin g Group Regional Fire Risk Assessment Model Based on AHP. Journal of Risk An alysis and Crisis Response, 6(1): 31-37.

[7] Wang W D, Qiao S, Zeng F L, Guo J, Hu ang C F. 2016. An Approach to Ranking I ntegrated Models for Risk Assessment by Using the Internet of Intelligences.Journa 1 of Risk Analysis and Crisis Response, 6 (1):2-7.

[8] Wang Z, Ge S S, Zhang R, Yang M Q, Ji ang D L. The Weight Analysis of Northea st Passage Straits Index Based on AHP an d Rough Set Theory. Proceedings of the 7 th Annual Meeting of Risk Analysis Coun cil of China Association for Disaster Prev ention (November 4-6, 2016, Changsha, China), pp.32-37.

[9] 李宇服,周俊,陈琦. 钦州港针地规划及针 抓力研究.中国水运,2009,9(09):11-13. 